\title{
Searches for BSM physics in dilepton, multilepton, and lepton+MET final states at CMS
}

\author{
Laurent Thomas*, on behalf of the CMS collaboration \\ University of Florida \\ E-mail: laurent.thomas@cern.ch
}

\begin{abstract}
We describe various searches for signs of physics beyond the Standard Model in proton-proton collisions collected by CMS in 2012 or 2015. These datasets correspond respectively to around $20 \mathrm{fb}^{-1}$ of integrated luminosity at a center of mass energy $(\sqrt{s})$ of $8 \mathrm{TeV}$ and around $2.5 \mathrm{fb}^{-1}$ at $\sqrt{s}=13 \mathrm{TeV}$. The analyses presented here focus on final states with one or several leptons and possibly missing transverse energy, for which the background from Standard Model processes is reduced, and primarily targets the production of new particles with masses in the $\mathrm{TeV}$ range. The performances of the lepton reconstruction and identification at large transverse momenta play a crucial role in these searches and are discussed.

No evidence of new physics is seen and upper limits level are set at the $95 \%$ confidence on the cross section of new phenomena leading to the various final states considered. The results are interpreted as lower limits on the mass of new particles predicted by a variety of models, leading to exclusion well above $1 \mathrm{TeV}$ in many cases, and surpassing the previous results at $\sqrt{s}=8 \mathrm{TeV}$ for all the analyses performed with the 2015 dataset.
\end{abstract}

XXIV International Workshop on Deep-Inelastic Scattering and Related Subjects

11-15 April, 2016

DESY Hamburg, Germany

\footnotetext{
*Speaker.
} 


\section{Introduction}

Despite its amazing successes, there exist strong reasons to think that the Standard Model (SM) consists of a low energy limit of a more fundamental theory which might lie at the $\mathrm{TeV}$ scale. Thanks to the high energy of its proton beams, the LHC makes it possible to probe this scale and the CMS collaboration [1] has studied a large variety of final states in the hope of observing an excess of events with respect to the SM expectation. Of particular interests are the purely leptonic final states, characterized by a SM background reduced by several orders of magnitude with respect to hadronic final states, as illustrated in figure 1 (left). In scenarios where lepton universality holds, the precise momentum measurement of electrons and muons together with their high selection efficiency and purity give to these channels the best sensitivity. Despite their challenging reconstruction, hadronically decaying taus are however of primary importance in models where enhanced tau production is expected.

After collecting data at $\sqrt{s}=8 \mathrm{TeV}$ in 2012, the LHC delivered its first proton proton collisions at $13 \mathrm{TeV}$ in the middle of 2015. Despite an integrated luminosity smaller than in 2012, the increased beam energy of the 2015 dataset would strongly enhance the production cross section of $\mathrm{TeV}$ particles, Figure 1 (right) which shows the parton luminosity ratio between 13 and $8 \mathrm{TeV}$ as a function of the parton-parton invariant mass suggests indeed that for the $13 \mathrm{TeV}$ dataset should surpass the $8 \mathrm{TeV}$ dataset sensitivity for masses greater than $2 \mathrm{TeV}$.

After a short discussion of the performances of the reconstruction of charged leptons in CMS with special emphasis on high transverse momentum (high $p_{T}$ ), this article presents five recent results obtained by CMS in searches for new physics with one or more leptons and possibly missing transverse energy $\left(E_{T}\right)$, of which four were obtained with the 2015 dataset.
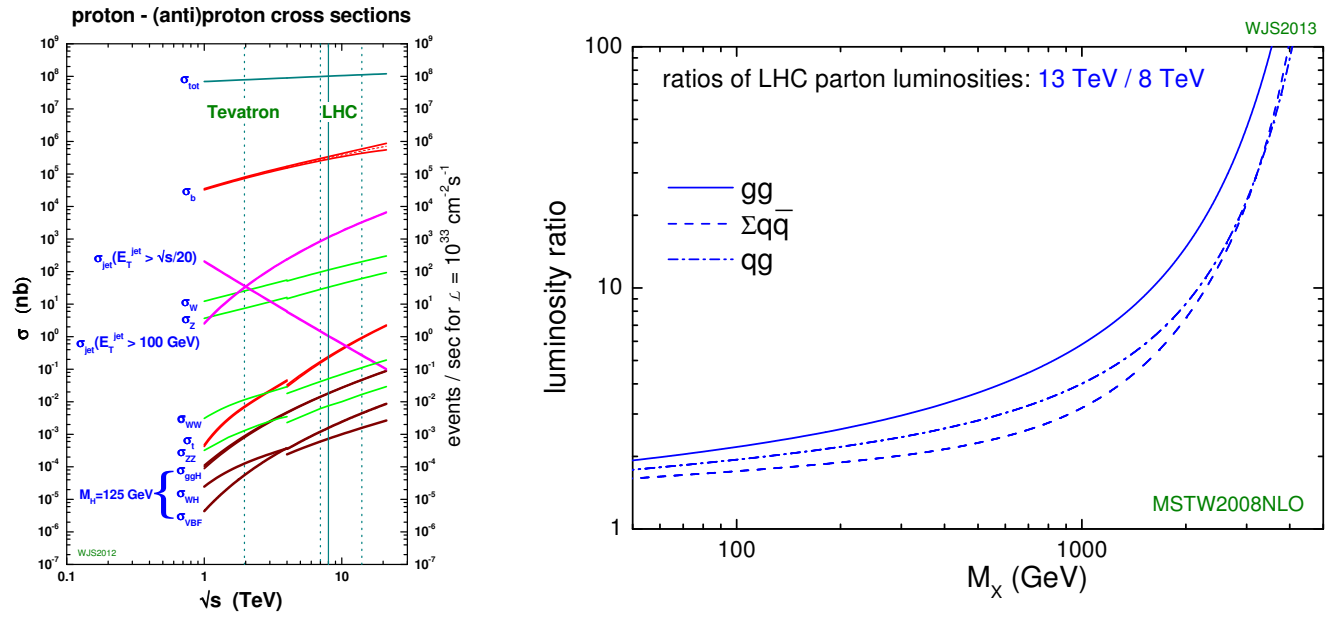

Figure 1: Left: cross section of various Standard Model processes in proton-(anti)proton collisions as a function of the center of mass energy. Right: Parton luminosity ratio between proton-proton collisions at 13 and $8 \mathrm{TeV}$ as a function of the parton-parton center of mass energy [2]. 


\section{Lepton reconstruction and performances in CMS}

The electron reconstruction starts from the association of an electromagnetic calorimeter (ECAL) deposit with a track. Various criteria on the track quality, track-cluster matching and cluster shape are then applied on the electron candidate. The electrons considered here are also required to be isolated. Figure 2 (left) shows the typical selection efficiency as a function of the electron $p_{T}$ for various pseudorapidity $(\eta)$ ranges, typically reaching values greater or equal to $80 \%$ for $p_{T}>100$ $\mathrm{GeV}$. For $p_{T}>100 \mathrm{GeV}$, the momentum measurement is fully driven by the ECAL and a resolution of $1.5 \%$ of the mass is reached for dielectron invariant masses greater than $1 \mathrm{TeV}$. A sizeable charge mismeasurement $(\geq 1 \%)$ is also expected. Finally, for extremely high energy deposits ( $>$ 1.7 TeV per ECAL cell in the barrel), the readout can saturate. In the analyses presented here, this saturation limit has however not been reached.

Muons tracks are primarily built by combining tracker and muon chambers hits. The selection efficiency is usually higher than in the electron case. It is shown as a function of the number of vertices in figure 2 (center) for $Z \rightarrow \mu \mu$ events. The momentum measurement relies only on the tracker for $p_{T}<200 \mathrm{GeV}$ while muon chambers also contribute for higher $p_{T}$. Because of the small curvature of the track, the $p_{T}$ resolution degrades as the $p_{T}$ increases. For a $1 \mathrm{TeV}$ dimuon resonance, the typical selection efficiency reaches $95 \%$ per muon and the mass resolution is $4 \%$.

Hadronically decaying taus are reconstructed using a specific algorithm looking for jets composed of one or three charged hadrons and up to 2 photons. As for light leptons, isolation criteria are applied. Figure 2 (right) illustrates the challenge of the tau reconstruction by showing the visible di tau invariant mass for events passing a standard $Z \rightarrow \tau \tau$ selection. The undetected neutrino(s) from the tau decay shifts the mass peak to values lower than the $Z$ pole mass, and the contribution from gluon or quarks jets misidentified as taus (QCD) is much larger than in $e e$ or $\mu \mu$ channels. The typical tau selection efficiency is also lower and reaches around $50 \%$ for $p_{T}>100 \mathrm{GeV}$.
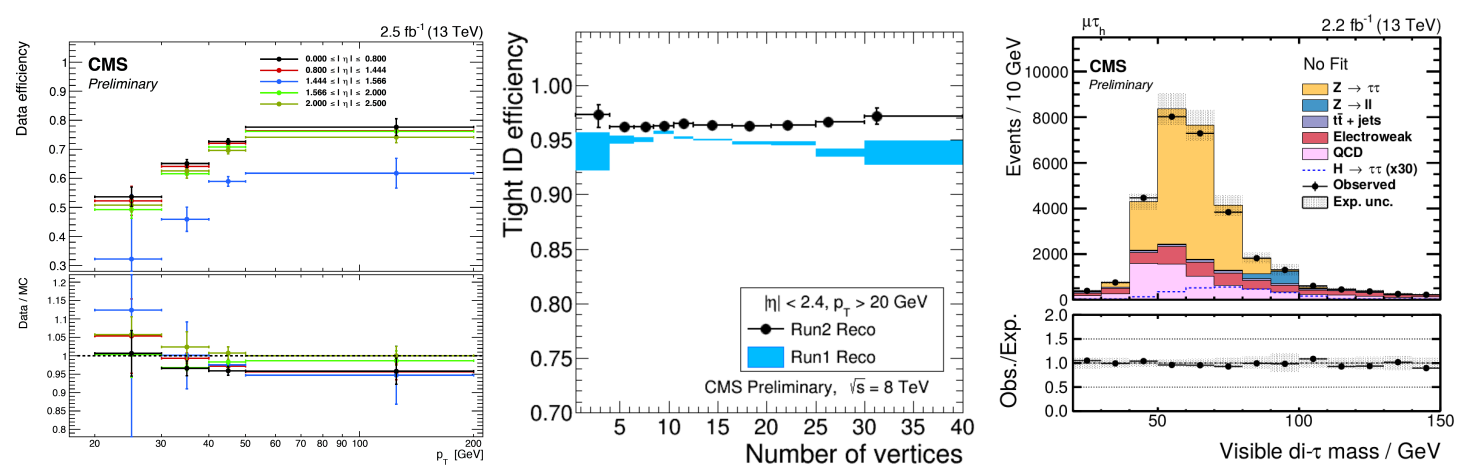

Figure 2: Left: Efficiency of a typical electron selection as a function of the electron $p_{T}$ for various $\eta$ regions [3]. Center: Efficiency of a typical muon selection as a function of the number of vertices [4]. Right: Visible mass of pairs of tau leptons in the muonic tau-hadronic tau final state [5].

\section{Search for narrow resonances in dielectron or dimuon final states}

The observation of a new resonance in the high mass tail of the dilepton invariant mass spec- 
trum would be a clear indication of new physics. A search for such a bump was therefore performed by CMS in the dielectron and dimuon channels with the first data collected at $13 \mathrm{TeV}$ [6]. The highest mass dilepton pair recorded in the dielectron channel is displayed in figure 3. No significant excess with respect to the SM background, dominated by the Drell-Yan process, was observed, and upper limits were set on $R_{\sigma}$ defined as the ratio of the production cross section times branching ratio of a new heavy resonance over the same quantity in the $Z$ peak region. The left plot of figure 4 compares the observed limits for various intrinsic widths of the new resonance while a comparison of the observed and expected limits is presented in the right plot. A lower limit of $3.15 \mathrm{TeV}$ is set on the mass of a heavy copy of the $\mathrm{Z}$ boson $\left(Z_{S S M}^{\prime}\right)$.

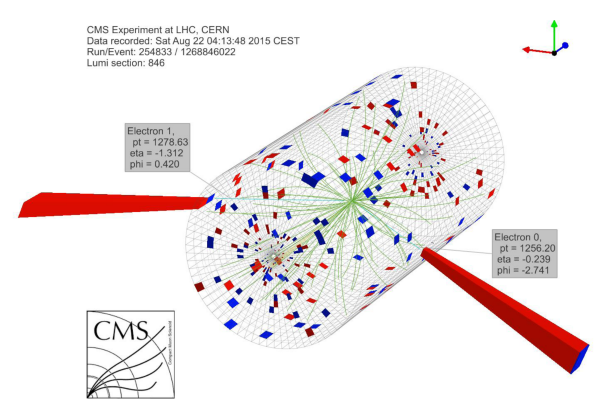

Figure 3: The highest mass dielectron pair event recorded by CMS in 2015, with a mass of $2.9 \mathrm{TeV}$.
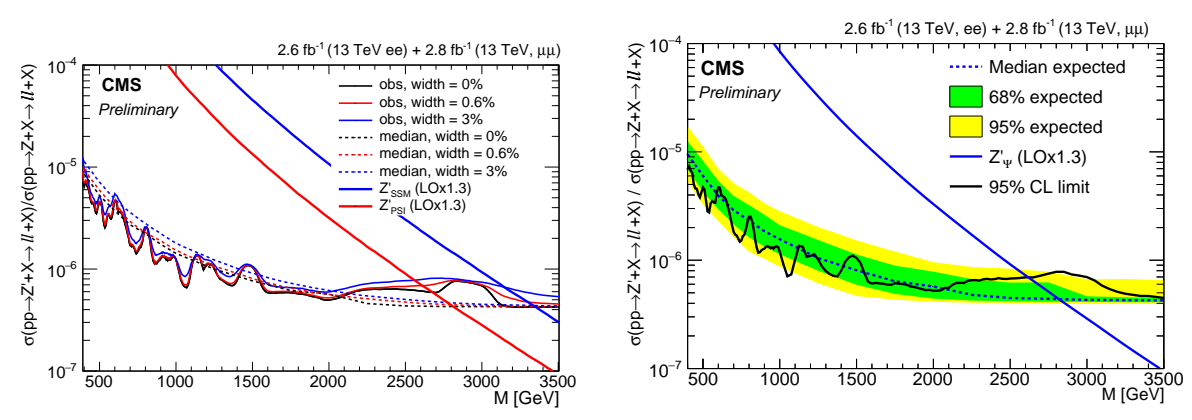

Figure 4: Left: Observed limits on $R_{\sigma}$ for various intrinsic widths of a dilepton new resonance. Right: Observed and expected limits on $R_{\sigma}$ for dilepton resonance with an intrinsic width of $0.6 \%$.

\section{Search for SSM $W^{\prime}$ production in the single lepton $+E_{T}$ final states}

The decay of a new heavy charged boson into a lepton and a neutrino was also investigated in the $13 \mathrm{TeV}$ dataset, in both $e$ and $\mu$ [7] and hadronic $\tau$ channels [8]. The event selection requires a high $p_{T}$ lepton and large missing transverse energy, well balanced in magnitude and in azimuthal angle. In both cases, a shape analysis is performed based on the distribution of the invariant mass of the lepton $p_{T}$ and the missing transverse energy $\left(M_{T}\right)$, shown in figure 5 for each lepton flavour separately. Since only part of the total mass is reconstructed, a new resonance will lead to a Jacobian peak in this distribution. The distribution is further smeared in the tau channel due to the 
energy carried by the tau neutrino. The $e$ and $\mu$ channels are combined to exclude a heavy copy of the $\mathrm{W}$ boson, $W_{S S M}^{\prime}$, below $4.4 \mathrm{TeV}$ while the $\tau$ channel leads to an exclusion below $3.3 \mathrm{TeV}$.

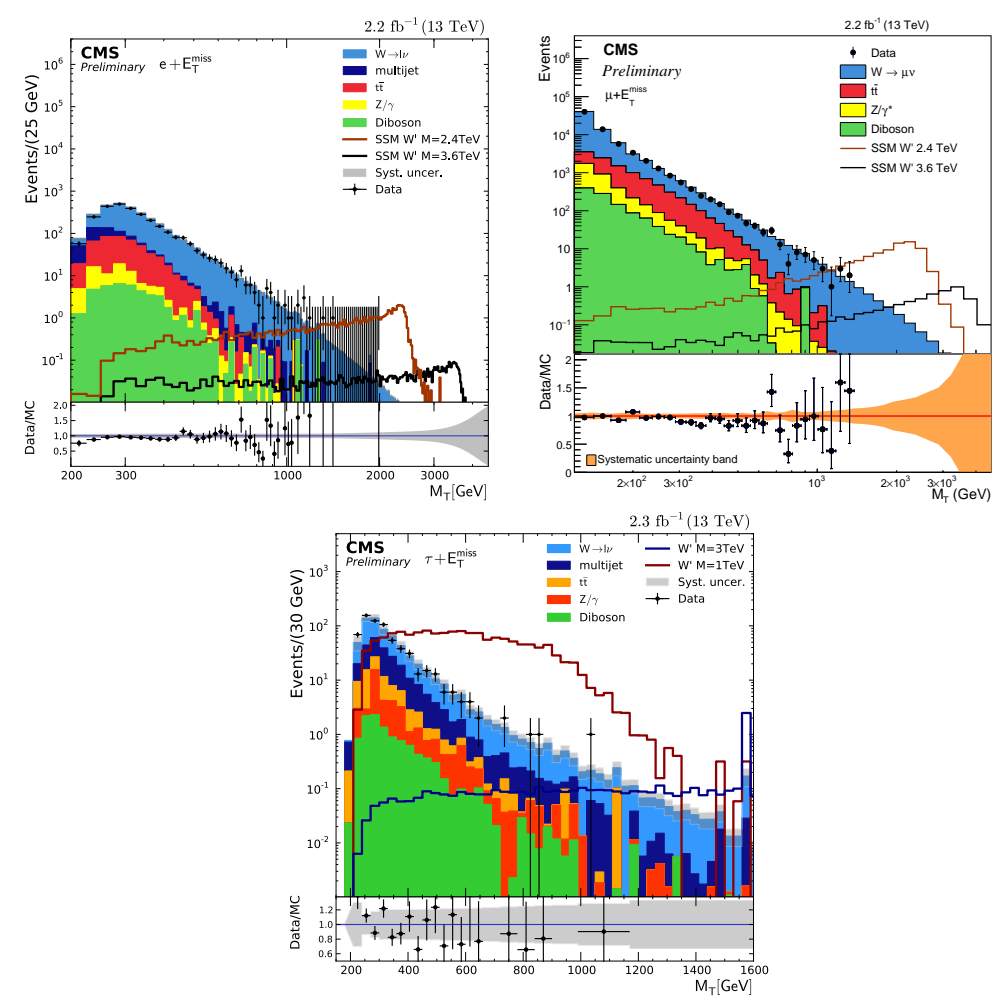

Figure 5: $M_{T}$ distribution of events selected in the single lepton + missing $E_{T}$ final state, for the $e$ (top left), $\mu$ (top right) and $\tau$ (bottom) channels, in data (black dots) and according to the SM prediction (full histograms). Also shown are the expected distributions for two $W^{\prime}$ with different masses.

\section{Search for Type-III Seesaw heavy fermions in the multilepton final state}

The 2015 dataset was also used to conduct a search for new physics with three leptons and missing transverse energy in the final state [9]. The benchmark model considered is the TypeIII SeeSaw model which provides an explanation of the neutrino masses by introducing massive charged $\left(\Sigma^{ \pm}\right)$and neutral $\left(\Sigma^{0}\right)$ fermion partners. At sufficiently high virtuality, a W boson could therefore decay into a $\Sigma^{ \pm} \Sigma^{0}$ pair as sketched in the left diagram of figure 6 .

The event selection starts from requiring three leptons (electrons, muons). Events are then classified according to the number of leptons, of opposite sign same flavour (OSSF) pairs and the invariant mass of these OSSF pairs (if any), as detailed in the right table of figure 6. They are further divided in 5 bins of $L_{T}+E_{T}$, where $L_{T}$ denotes the scalar sum of the leptons $p_{T}$. Since these regions are not expected to improve the signal sensitivity, the final selection rejects events where $L_{T}+E_{T}<350 \mathrm{GeV}$ and events where at least one OSSF pair is found but none of them has an invariant mass greater than $81 \mathrm{GeV}$. 
The observed and expected $L_{T}+E_{T}^{\prime}$ distributions are shown in figure 7 for two signal regions. No evidence of new physics is observed and, assuming an equal mass for the charged and neutral new leptons, a mass below $440 \mathrm{GeV}$ is excluded.

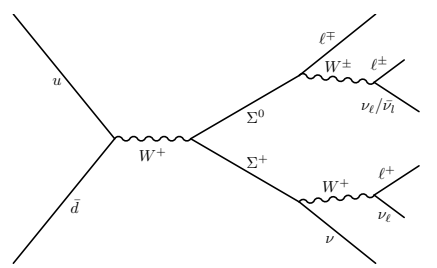

\begin{tabular}{|cccc|}
\hline Signal region & \# leptons & \# OSSF pairs & $M_{\text {OSSF }}(\mathrm{GeV})$ \\
\hline A & $=3$ & $\geq 1$ & $\geq 1$ pair $\in(81,101)$ \\
B & $=3$ & $\geq 1$ & lowest $M_{O S S F}>101$ \\
C & $=3$ & $=0$ & $/$ \\
D & $=4$ & $\geq 1$ & $/$ \\
\hline
\end{tabular}

Figure 6: Left: Feynman diagram of the production and decay of a $\Sigma^{+} \Sigma^{0}$ pair in the Type-III Seesaw model. Right: definition of the various signal regions used in the search for such a signal.
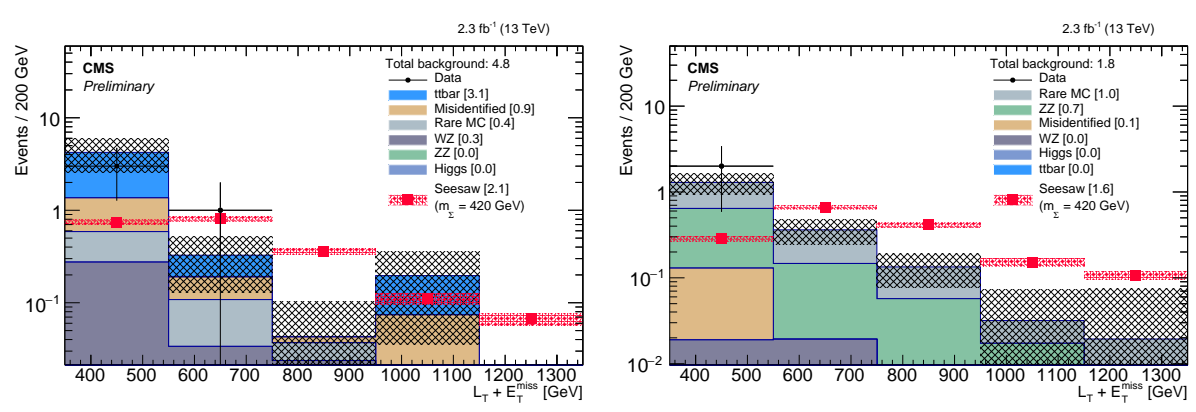

Figure 7: Distribution of $L_{T}+E_{T}$ in signal regions C (left) and D (right) defined above, for the 2015 data and for the SM backgrounds, together with the yields for a signal where the mass of the $\Sigma^{ \pm}$and $\Sigma^{0}$ is set to $420 \mathrm{GeV}$.

\section{Search for leptophobic $Z^{\prime}$ decaying into four leptons in the final state}

The results of the search for a new heavy resonance decaying into four charged leptons (electrons or muons) with the 2012 dataset have recently been released by CMS [10]. Such a resonance could be the signature of a leptophobic $Z^{\prime}$ decaying into two neutral intermediate particles, $\phi$, both decaying leptonically (figure 8, top). This generic analysis seeks for a narrow bump in the four lepton invariant mass spectrum. No condition is made on the number of electrons and muons and the $е e е \mu$ and $е \mu \mu \mu$ final states are also considered. In the case of an even number of electrons and muons, events with a same flavour opposite sign pair compatible with the $Z$ boson mass are rejected in order to suppress the dominant $Z Z$ background. If the mass of the intermediate particle is small, the two decay products can be emitted close to each other and a special treatment is therefore applied to reconstruct pair of electrons that could share the same ECAL deposit.

Figure 8 (bottom left) presents the mass spectrum observed in the data and predicted by the SM. A good agreement is observed and upper limits on the cross section are shown as a function of the resonance mass in figure 8 (bottom right), excluding a leptophobic $Z^{\prime}$ below $2.5 \mathrm{TeV}$ for $m_{\phi}=$ $50 \mathrm{GeV}$. 


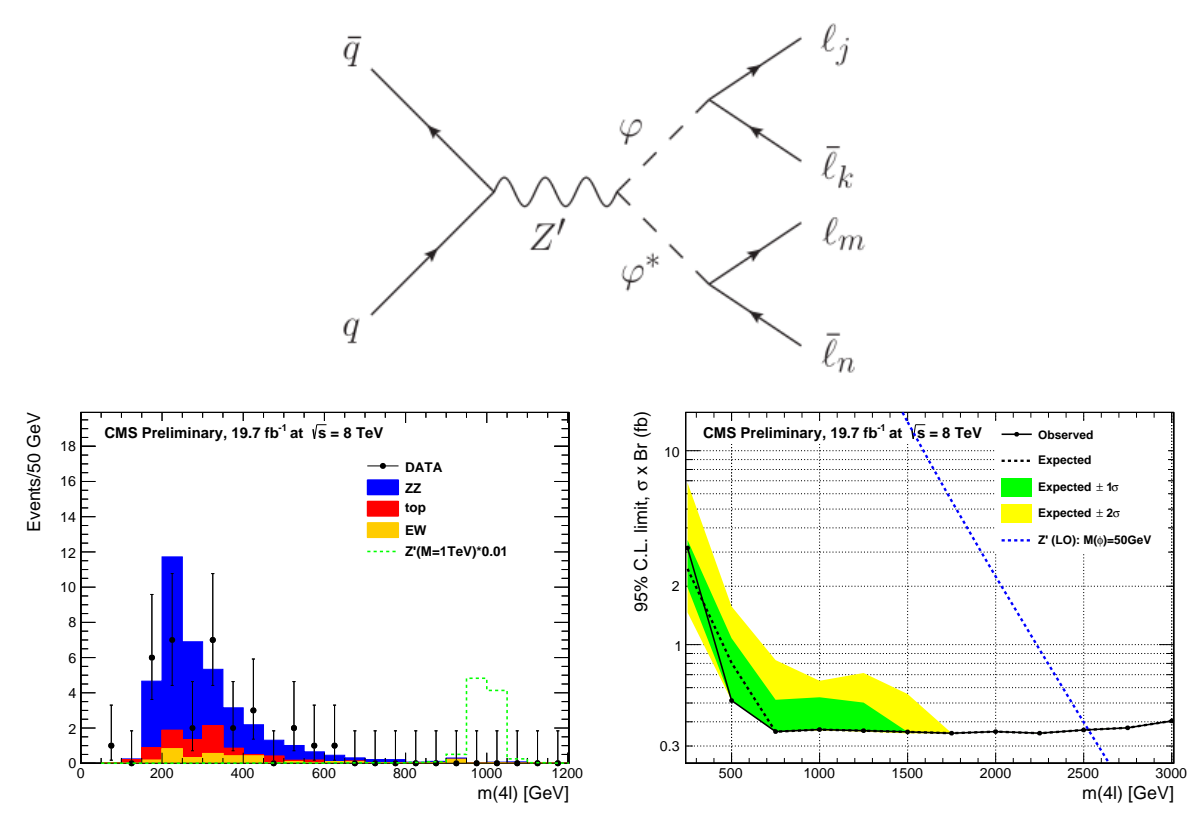

Figure 8: Top: Feynman diagram of the production and decay of a leptophobic $Z^{\prime}$. Bottom left: Four lepton invariant mass distribution in data, according to the SM prediction and for a $1 \mathrm{TeV}$ resonance. Bottom right: Upper limits on the cross section times branching ratio of such a resonance.

\section{References}

[1] The CMS Collaboration, The CMS experiment at the CERN LHC, JINST 3:S08004,2008.

[2] W. G. Stirling, Parton luminosity and cross section plots, http://www.hep.ph.ic.ac.uk/ wstirlin/plots/plots.html

[3] The CMS Collaboration, Electron and Photon performance using data collected by CMS at $\sqrt{s}=13$ $\mathrm{TeV}$ and 25ns, CMS-DP-2015-067.

[4] The CMS Collaboration, Muon Reconstruction and Identification Improvements for Run-2 and First Results with 2015 Run-2 Data, CMS-DP-2015-015.

[5] The CMS Collaboration, HIG PAG DPS plots for presentations before Moriond 2016,CMS-DP-2015-066.

[6] The CMS Collaboration, Search for a narrow resonance produced in $13 \mathrm{TeV}$ pp collisions decaying to electron pair or muon pair final states, CMS-PAS-EXO-15-005.

[7] The CMS Collaboration, Search for SSM W' production, in the lepton+MET final state at a center-of-mass energy of $13 \mathrm{TeV}$, CMS-PAS-EXO-15-006.

[8] The CMS Collaboration, Search for W' decaying to tau lepton and neutrino in proton-proton collisions at $\sqrt{s}=13 \mathrm{TeV}$, CMS-PAS-EXO-16-006.

[9] The CMS Collaboration, Search for type-III seesaw heavy fermions with multilepton final states using $2.3 \mathrm{fb}^{-1}$ of $13 \mathrm{TeV}$ proton-proton collision data, CMS-PAS-EXO-16-002.

[10] The CMS Collaboration, Search for leptophobic Z' decaying into four leptons in the final state at $\sqrt{s}$ $=8 \mathrm{TeV}$, CMS-PAS-EXO-14-006. 